\title{
Level of Oral Hygiene Practices and its Association with Socio-Demographyic Characters Among Middle School Students in Low Income Countries
}

Tecleab Okubai Gebregiorgis ( $\square$ teclinoo19855@gmail.com )

Department of Nursing,Orotta College of Medicine and Health Sciences(OCMHS),Asmara,Eritrea.

Alhassen Saleh Humed

Ministry of Health (MOH),Asmara,Eritrea.

Beraki Mael Tekle

Ministry of Health (MOH),Asmara,Eritrea.

Bereket Tesfamichael Ghebremedhin

Ministry of Health (MOH),Asmara,Eritrea.

Letebrhan Habtemariam Haile

Ministry of Health (MOH),Asmara,Eritrea.

Research Article

Keywords: Oral Hygiene, Practice, Middle School Children, Low Income Countries

Posted Date: July 7th, 2021

DOl: https://doi.org/10.21203/rs.3.rs-627216/v1

License: (c) (i) This work is licensed under a Creative Commons Attribution 4.0 International License.

Read Full License 


\title{
Level of Oral Hygiene Practices and Its Association with Socio-Demographic Characters among Middle School Students in Low-Income countries.
}

\author{
Authors \\ - Tecleab Okubai Gebregiorgis, (Email: teclinoo19855@gmail.com) $)^{\mathbf{1}^{*}}$ \\ - Alhassen Saleh Humed, (Email: bisratm100@ gmail.com) ${ }^{2}$ \\ - Beraki Mael Tekle, (Email:drklitot@gmail.com) ${ }^{2}$ \\ - Bereket Tesfamichael Ghebremedhin, (Email: abrahabrhanee@gmail.com) ${ }^{2}$ \\ - Letebrhan Habtemariam Haile, (Email: fanuel19855@gmail.com) ${ }^{2}$ \\ Authors Affiliation \\ ${ }^{1}$ Department of Nursing, Orotta college of Medicine and Health Sciences (OCMHS), \\ Asmara, Eritrea. \\ ${ }^{2}$ Ministry of Health (MOH), Asmara, Eritrea. \\ * Corresponding Author: Tecleab Okubai Gebregiorgis, Email: teclinoo19855@gmail.com
}

\begin{abstract}
Background: The practice of keeping the mouth clean to prevent common oral health diseases is necessary, especially in children who live in developing countries. This study aims to assess middle school students' level of oral hygiene practice (OHP) and its association with sociodemographic factors among children in low- income countries.

Methods: Descriptive cross-sectional study was conducted to determine level of OHP among Middle school students age of 12-15years. Data was collected from all participants $(\mathrm{N}=572)$ through face -to- face interview using a pretested and semi structured questionnaire. Pearson's chi-square test and multiple binary logistic regressions were used to analyze the whole data. Results: Overall, majority (70\%) of middle school children were having poor OHP. From the total respondents, only 201 (35.2\%) of the middle school children cleaned their teeth two times or more a day,16 (3.1\%) used dental floss for dental cleaning and 314 (54.9\%) brushed their teeth after meals. The most utilized oral hygiene tools were toothbrush 554.8 (97\%), chew-stick 411.3(71.9\%), and toothpick 192 (33.6\%). Most of the participants 554(97.4\%) used toothpaste during tooth cleaning, but 509 (89\%) of the children were unaware about the presence of fluoride in their toothpaste. From the children, 196 (34.4\%) changed their brush every three months, and only 72 (12.6\%) applied circular strokes while brushing their tooth. The most frequently consumed sugar foods and drinks were bread and biscuits, sugar containing chewing gum and tea cited as 536(93.7\%), 256(54.8\%) and 486(85\%) of the respondents respectively.
\end{abstract}


Around half (52\%) of the respondents didn't cleaning their tongue at all. Only $112(19.5 \%)$ visited a dentist during the past twelve months. OHP score was significantly different for gender and school type $(\mathrm{P}<0.05)$. Females children were having better OHP than males $\mathrm{AOR}=0.636$ $(0.441-0.918, \mathrm{P}=0.012)$. Moreover, Arbate Asmara was less likely to have poor OHP than Bana AOR=0.426 (0.197 - 0.92, P=0.030). Conclusions: This study found that, majority of the children was having poor OHP. Sex and school of middle school children in low income countries had a significant correlation with OHP. The results of this study indicate the need of a Comprehensive oral hygiene practice education for the children and their parents to achieve good oral hygiene in the middle school.

Key words: Oral Hygiene, Practice, Middle School Children, Low Income Countries

\section{Background}

Good oral hygiene is the foundation of a healthy mouth and prevents $80 \%$ of all dental problems

[1]. The practice of keeping the mouth clean to prevent common oral health diseases is necessary, especially in children who live in developing countries [12]. It is known that oral hygiene (Greek, hygienos) is the condition or practice of maintaining the tissues and structures of the mouth in healthy state [23]. Children and adolescents who develop good oral hygiene practices were more likely than others to maintain these healthy behaviors in adulthood. Children in low- and middle-income countries (LMICs) may have limited access to some of these tools in addition to lacking access to routine dental care [31,32].

WHO Defined oral health as "a state of being free from chronic mouth and facial pain, oral and throat cancer, oral sores, birth defects such as cleft lip and palate, periodontal gum disease, tooth decay and tooth loss, and other diseases and disorders that affect the oral care" [34]. There are various environmental and life style factors such as nutritional status, tobacco smoking, alcohol, poor oral hygiene, stress, and systemic conditions linked to the oral diseases [12]. Oral and dental hygiene is essential to good health in general and preventing occurrence of many diseases [35]. Dental caries and periodontal problems are due to poor oral hygiene practices [36]. Previous study findings indicate that the best way to avoid oral disease and tooth decay is through promotion of oral hygiene practices [32]. The development and implementation of preventive programs is necessary to introduce healthy lifestyle and self-care practices, especially for the children of these countries [2]. 
Oral hygiene maintenance may be a totally ignored practice in people of the low socioeconomic class of developing countries [3]. Studies indicate that $90 \%$ of the students have experienced dental cavities and could lead to tooth loss at a very young age [2]. Oral health promotion through schools is recommended by WHO for improving knowledge, attitude, and behavior related to oral health and for prevention and control of oral health problems among school children $[4,5]$.

Although literature regarding OHP is abundant, but little is known among middle school children's practice in low income countries with association to socio demographic factors. In Eritrea, Dental caries was ranked as the highest morbid disease in the years 2013 and 2014 and second highest reported disease during the years 2011, 2012, 2015 and 2016. Dental caries was found to be a common public health problem among 12 years old Eritrean than other developing countries. Gaps in dental health service utilization, dental health practices and suboptimal water fluoride levels contribute to poor dental health among school children in Eritrea [8]. Therefore, this study report findings regarding middle school children's level of OHP and its association with socio-demographic factors in low income countries, Eritrea.

\section{Methods}

\section{Study design and sample size}

Descriptive, cross-sectional design was applied to assess level of OHP among (N=572) middle school children in low income countries Asmara, Eritrea.

\section{Study Setting and population}

This study was carried out in the middle schools of capital city of Eritrea, Asmara, a more populated and consisting of all the nine ethnicities located in the other region. The population of Eritrea (as of 2019) was estimated about 3,497,117. Middle school is one of the governmental institutions at secondary school level that begun its service since the independence, 1991 servicing from 12-15 years old children.

The emphasis of this study was on middle school students $\left(6,7\right.$ and $8^{\text {th }}$ grades) from the age of 12-15 years old. After checking Inclusion and exclusion criteria a multi stage random sampling technique was used to recruit participants. Data was collected by the researchers from February to June 2019 through face to face interview with a semi-structured questionnaire in the four randomly selected schools. Finally, from 580 targeted study populations, only $572(98.6 \%)$ questionnaires were found to be correctly filled and entered for data analysis in the SPSS. 


\section{Data collection tools and procedure}

The tool and its scoring system was adopted and modified by the researchers to suit the study design \&setting after reviewing similar studies conducted worldwide especially in developing countries by $[9,34,11]$. First, a preliminary draft of the survey used in the main research was distributed to a panel of colleagues, dental therapists and lecturers at OCMHS, by providing the study guiding and research questions. Next, based on their comments and feedback it was examined if the used tool can assess level of OHP and its association with socio-demographic characters by piloting for an appropriate correction \& modifications to be made for its content and clarity according to the study objective.

Moreover, in order to reduce the data entry errors, a Census and Survey Processing System (CSPro, Version 7.0) software package entry program was used, that automatically checks the data structure of the file using skip patterns and range of valid values. Finally, after the pilot study was conducted, reliability coefficient and normality was checked, necessary modifications were made to the questionnaire and finally used for this study data collection.

\section{Variable scoring system}

The Research questionnaire has two parts that include socio-demographic and practice variables.

\section{A. Socio-demographic information section}

This section includes twelve items such as the participants' school type, age, sex, and grade, place of residence, ethnicity, family size ,religion, fathers' and mothers' occupational and educational status summarized as frequency, percentage, $(\mathrm{M} \pm \mathrm{SD})$.

\section{B. Oral hygiene practice section}

In order to measure the level of OHP and its association with socio-demographic characters of the middle school children's sixteen questions were used. Each correct response was given one mark and zero score to an incorrect and none response question. The composite practice scores were totaled from 16 questions giving an ideal minimum and maximum score of 0 and 65 respectively. To have better interpretation the oral hygiene practice was expressed in percent form, as believing score increase and practice also.

\section{Data Analysis Plan}

Data analysis was carried out using both descriptive and inferential statistics with the help of Statistical Package for Social Sciences (SPSS version, 22). After completion of recoding and entering the raw data to SPSS the data was cleaned and normality of the data was checked and 
accordingly an appropriate statistical analysis was selected. Frequencies, proportions and descriptive statistics (Mean \pm standard deviation) were used to describe the data. Moreover, to inferential statistics like Pearson's chi-square test, binary \& multiple binary logistic regressions were used to assess the significance of difference and to find correlation between OHP and socio-demographic characteristics respectively. The statistical significance was set at $95 \%$ confidence interval and $\mathrm{P}$ value $<0.05$ level. Finally, the result was summarized in the form of graphs, charts and tables.

\section{Operational definition}

Level of Oral hygiene practices: This is an activity undertaken by the children in order to protect, promote, or maintain oral health, prevent dental diseases and bad breath. The practices include tooth brushing, tongue cleaning, tooth and tongue cleaning tools, flossing, mouth rinsing, and dental visit and reducing sugar consumptions.

Good oral hygiene practice: This is cleaning mouth, teeth and tongue (oral cavity) using the proper tool, technique and timing among middle school children, measured by the proportion of question responded correctly by the participants.

\section{NUMBER OF CORRECT ANSEWER x 100}

\section{TOTAL NUMER OF QUESTIONS}

Poor oral hygiene practice: This is either improper use of tool, technique or timing to maintain oral health among school children, measured by the proportion of question responded incorrectly by the participants.

\section{MBER OF IN CORRECT ANSEWER x 100}

\section{TOTAL NUMER OF QUESTIONS}

Associated factors: These are socio-demographic variables that influence oral hygiene practice among middle school students either by facilitating or hindering it.

Middle school: An intermediate school between elementary and high school which encompasses from grade 6 up to 8 th.

Students: These are 12 to 15 years old middle school children who are enrolled in formal academic middle schools.

Low income countries: According to the World Bank, low-income countries are nations that have a per capita gross national income (GNI) of less than $\$ 1,026$. 


\section{Validity and reliability}

The face and content validity of the questionnaire was guaranteed through reviewing the English and Tigrigna version by experts in a panel at the Department of Nursing and Dentistry at Orotta College of Medicine and Health Sciences. In order to confirm the construct validity of the survey used for this study, the questionnaire was based on similar standardized surveys used in previous studies regarding middle school students $[34,9,11]$. The questionnaire was tested for reliability by computing the Cranach's alpha for Practice $(r=0.726)$.

\section{Pilots Testing}

A pilot study was conducted in a different middle school (Fithi) Schoolin Asmara, among 115 (10\% of sample size) children in order to assess the feasibility of the study, validity, sensitivity and understandability of the questionnaire used by researchers.

\section{RESULTS}

\section{Socio-demographic characteristics of the respondents}

A total of 580 study participants were eligible, and 572 (98.6\%) of them were enrolled in the Study. This study revealed 300(52.4\%) of the participants were males and 272 (47.6\%) were females, the mean age was( $\mathrm{M}=13.03 \pm 0.986 \mathrm{SD})$. Majority of the respondents, $539(94.2 \%)$ were Christians by religion, 534 (93.4\%) were from urban residents and 356 (62.2\%) mentioned that their family size falls in the category of more than 6 members. As to the parental occupational status, $243(42.5 \%)$ reported that their father's as being governmental employee, $359(62.8 \%)$ mothers as being housewife, 231 (40.4\%) cited their paternal educational status as being primary level or below and $265(46.3 \%)$ their mother's educational level as being primary level or below (Table 1).

\section{Oral Hygiene Practice of the Middle School Children}

For this study, only $201(35.2 \%)$ of the students cleaned their teeth two times or more a day, whereas $128(22.4 \%)$ cleaned once a day (Fig.1).

\section{Tooth cleaning tools used by the participants}

The Most commonly used tooth cleaning tools were toothbrush and local Chew-stick utilized by $555(97 \%)$ and $411(71.9 \%)$ of participants respectively (Fig.2). However, only 16 (2.8\%) used dental floss for dental cleaning and only one performed dental floss once a day. Moreover, $192(33.6 \%)$ used tooth picks and $22(3.8 \%)$ used other tools like match sticks and piece of cloth 
for tooth cleaning. Majority of the participants (97.4\%) used toothpaste during tooth cleaning, from those only $40(7.2 \%)$ used fluoride containing toothpaste, but 509 (89\%) were unaware about the presence of fluoride in their toothpaste (Table.2). Out of the students who used toothpaste, only 164 (29.4\%) applied toothpaste to full length of the bristles. Regarding the frequency of changing toothbrush, out of 555 only 196 (35.3\%) changed their brush every three months. In addition, more than one third (36.4\%) of them changed their brush when bristles get frayed up (Table.2).

More than half of the respondents $(55.1 \%)$ brushed their teeth after meals, $89(15.6 \%)$ brushed before meals, whereas only $117(20.5 \%)$ brushed their teeth both before and after meals and about $51(8.9 \%)$ brushed at any convenient time. Concerning to the length of brushing time, more than half of the respondents $(62.2 \%)$ brushed their teeth for two minutes or more, more than one quarter $(28.2 \%)$ brushed for one minute or less and $55(9.6 \%)$ were unaware about the time they elapse to brush their teeth (Table.2).

\section{Oral Hygiene Practice of study participants}

In the present study, about one quarter of the respondents (26.6\%) applied horizontal strokes and $57(10 \%)$ applied vertical strokes while brushing. Furthermore, about half (50.9\%) of the respondents applied both techniques, whereas only 72 (12.6\%) applied circular strokes during brushing their teeth. About 268 (46.9\%) of the students rinsed their mouth always, 252 (44.1\%) rinsed sometimes, however, $52(9.1 \%)$ never rinsed their mouth after each meal (Table.2).

\section{Prevalence of tongue cleaning among participants}

Regarding tongue cleaning, 280 (49\%) of the respondents cleaned their tongue with different tools (Fig. 3). Out of those only 26 (9.3\%) used tongue cleaner, 208 (74.3\%) used toothbrush, 29 $(10.3 \%)$ used their fingers and $25(8.9 \%)$ used other materials like salt and chew-stick.

\section{Frequency of consumed sugary foods and drinks}

With regard to the frequency of eating sugary food and drinks, the most frequently consumed were bread and biscuits, sugar containing chewing gum and tea which cited by 552 (96.5\%), 263 (46\%) and 509 (89\%) respondents respectively (Fig.4).

\section{Frequency of dental visit of the participants}

In the current study, only $112(19.5 \%)$ of the participants visited a dentist during the past twelve months, $49(8.5 \%)$ visited two times or more and $63(11 \%)$ visited once. About 114 $(20 \%)$ didn't visit a dentist in the past twelve months, whereas more than half $(60.5 \%)$ never visited a dentist (Fig.5). 


\section{Reason for dental visit among participants}

Out of those who visited a dentist $102(91.1 \%)$ visit was due to pain or problem with teeth, 9 (8\%) for treatment or follow-up and only one for routine check-up of teeth or cleaning (Fig.6).

\section{Level of Oral Hygiene Practice among Middle School Children}

The overall practice among the respondents was poor in which $69.6 \%$ was found to have poor oral hygiene practice and only $30.4 \%$ had good practice (Fig.7).

\section{Factors associated with oral hygiene practice}

The oral hygiene categories such as poor and good practice were used to identify the association with the socio-demographic variables. After checking normality of the variables, chisquare and binary logistic regression were used to identify the association of socio-demographic characteristics with oral hygiene practice.

Both sex $\left(\chi^{2}=7.711, \mathrm{P}=0.005\right)$ and school type $\left(\chi^{2}=14.578, \mathrm{P}=0.012\right)$ variables had significant association with oral hygiene practice score. This can inferred as Arbaete Asmara and Bahtimeskerem had better oral hygiene practice, whereas Hadnet and Bana has higher proportion of poor oral hygiene than the other type of schools, but others had similar OHP score. However, there was no statistical significant score between OHP and the other children socio-demographic variables $(\mathrm{P}>0.05)$ (Table.3).

\section{Multivariate binary logistic regression}

The result of binary logistic regression indicates that there was significant association between practice and sex, $\mathrm{AOR}=0.636(0.441-0.918, \mathrm{P}=0.016)$, females were 0.636 times less likely to have poor practice than males. Regarding the association between practice and school type, Arbate Asmara and Bana, AOR = 0.426 (0.197 - 0.920, P=0.012), were found to be different. Therefore, there was a significant association between practice and the above schools; those who are from Bana middle school are 0.426 times more likely to have poor oral hygiene practice than those who are from Arbate Asmara (Table.4).

\section{DISCUSSION}

Oral hygiene is an important aspect of the health to be discussed in middle school children, especially in developing countries. Proper oral hygiene practice among children can be influenced by its environment such as family, school and community as well. So, empowering children, parents and community with standardized practice is a paramount to have a good oral health. Therefore, regular oral hygiene education should be incorporated to school curriculum 
and community paradigms accordingly. This will promote and improve oral hygiene practices among children by increasing awareness. This current study was aimed at assessing level of OHP and its association with socio demographic factors among middle school students in low income countries, Eritrea. It also involved 572 middle school students from four selected subzones of Asmara with a response rate of $98.6 \%$.

In the current study, $52.4 \%$ of respondents were males and $47.6 \%$ were females similar with a studies done $[16,18,19]$. The mean age of the respondents in this study was 13.03 comparable with a studies done $[19,20]$, were most of the participants, $56.6 \%$, reported that their family size falls in the category of 5 to 7 members, consistent with a study done [21]. About $62.8 \%$ reported that their mothers being housewife lower than study done [22]. Only 30.6\% reported that their father's education as secondary level and $44.6 \%$ reported their mother's education as secondary level. On the contrary, a study done [22] reported higher parental educational level being graduate or postgraduate.

\section{Level of Oral hygiene practice}

Level of Oral hygiene practice among the respondents was assessed by using different related questions. Majority of the participants (70\%) had poor practice, similar to [22]. In this study, more than one third of the children 201 (35.2\%) cleaned their teeth two times a day or more, whereas 128 (22.4\%) cleaned once a day. In a study conducted [10] was similar for those who brushed twice a daily, whereas more than half cleaned once daily higher than the present study. On the other hands a study done [19] indicated slightly higher than the current study. Moreover, a studies done $[32,16,21]$ was higher than the results of this study. This difference could be due to the difference in the economic status and knowledge of the children and parents, and dental services including oral education access in the different study setting.

The most Commonly utilized oral hygiene tools by the children were toothbrush (97\%), chewstick (71.9\%), toothpick (33.6\%) and thread (2.8\%). A similar study done [21] and [26]. Moreover, a study conducted [5] showed a similar proportion utilized dental floss and toothbrush with toothpaste, however a higher proportion used toothpick. In a previous study done in Eritrea [8] among 12 years old school children similar proportion used local chew stick, whereas lower proportion used toothbrush. Nevertheless, a study done in Nigeria reported lower utilization of chew stick as tooth cleaning material. Chew-stick was highly utilized in developing countries that could be due low socio-economic status, being easily accessible and its frequent use by the communities. The use of floss was negligible in this study, attributed to the lack of knowledge 
regarding the importance and technique of flossing that indicated the limited access of dental services in Eritrea.

Out of those who used toothbrush $35.3 \%$ changed their brush every three months, whereas $36.4 \%$ changed when bristles get frayed up similar to study done [25]. On the contrary a study [22] revealed lower than this study changed their brush within three months. An inadequate knowledge of the children \& parents and economic barriers could affect the interval of changing brush.

Study done on Oral hygiene practice among the primary school children in selected rural areas of Bangladesh in 2012 shows that oral hygiene practices, in particular, the daily tooth cleaning without tooth brush was high $(63.8 \%)$ and tooth brush user $(33.2 \%)$. Most of the respondents reported brushing on daily basis more at the morning (76.8\%) and two times $23.2 \%$ and this may be one of the factors for high prevalence of poor oral hygiene as depicted by the presence of plaque, calculus, caries, gingival bleeding in this study population [15].

The toothbrush with toothpaste was the most common oral hygiene aid used for cleaning teeth and it was observed that a greater number of students brushed their teeth twice daily [30].

Majority of the respondents $(97.4 \%)$ used toothpaste during tooth cleaning, but only $7.2 \%$ of the respondent used fluoride containing toothpaste and most of the $(89 \%)$ were unaware about the presence of fluoride in their toothpaste. Similarly, in a study done [25] used toothpaste while brushing, but much higher than this study finding used fluoride containing toothpaste. Similar studies done $[8,9,20]$ showed discrepancies on the use of tooth cleaning as the current study that could be due to the limited knowledge regarding fluoride use by the respondents. Out of the students who used toothpaste, only $29.4 \%$ applied toothpaste to full length of the bristles, which was less than that of $[7,20]$. Increased report of toothpaste utilization in this study was due to annual provision of toothpaste by the schools. However, small proportion cleaned their teeth frequently, because the schools in Asmara provide one tooth-paste for each student yearly and after the completion of that only $62.8 \%$ were provided with toothpaste by their parents.

With regard to the duration of brushing, $62.2 \%$ brushed their teeth for two minutes or more, consistent with a study done in Chandigarh, India, 2013 [12] (64.1\%). In addition, a study conducted in Madagascar reported lower (44.3\%), whereas in Sarawak, Malaysia, higher results (95.7\%) were reported [27] respectively.

In the present study, more than half $(50.9 \%)$ of the respondents applied both horizontal and vertical strokes, whereas only $12.6 \%$ applied circular strokes during tooth brushing. A similar 
finding studied [7]. But, [20] reported relatively higher proportion of circular technique and combination of the other three techniques.

Less than half (46.9\%) of the students always rinsed their mouth, whereas $9.1 \%$ never rinsed their mouth after each meal an inconsistent result reported [16, 26]. Regarding tongue cleaning, only $49 \%$ of the respondents cleaned their tongue from which $74.3 \%$ utilized toothbrush, whereas only $9.3 \%$ utilized tongue cleaner, are commended tool, lower than study found [7]. On the contrary to the current study [7] reported that none used toothbrush for tongue cleaning. This low practice of tongue cleaning could be due to the lack of knowledge regarding its importance. So, increasing children's awareness through oral education in school or visiting by professional team could improve their practice.

The most frequently (several times a week or more) consumed foods and drinks were bread and biscuits, sweets and candy, and soft drinks cited as $96.5 \%, 25.6 \%$, and $21.8 \%$ of respondents respectively. A study done in Iran [12] reported different result than this study, but study done [27] reported higher than this study. Children who live in urban areas like Asmara were more accessible to sweet foods such as bread and cakes which were frequently consumed as part of their regular diet. So, empowering children and parents with the knowledge of the cariogenic effect of these foods and the importance of cleaning following each meal will reduce the risk of oral diseases.

In the current study, only $19.5 \%$ of the participants visited a dentist during the past twelve months, whereas more than half $(60.5 \%)$ never visited a dentist. A different result was reported $[3,21]$, but similarly a study conducted [20] revealed regularly visited a dentist, however, lower proportion never visited a dentist as compared to this study. Furthermore, [20] found that, the reason for the visiting was tooth ache being lower and routine check-up being higher than this study A similar study conducted in Asmara, Eritrea [8]. This unsatisfactory utilization of dental services could be due lack of accessible dental services, inadequate knowledge, or low economic status that decreases demand of the services. Integrating oral health education into school curriculum will increase awareness among children and giving attention to proper utilization by providing affordable and accessible services which could increase dental visits and thereby improving oral hygiene practices and reducing oral diseases [3].

\section{Factors associated with level of oral hygiene practice}

In this study cited, Sex and school type of the middle school children in low income countries have significant association with OHP. A significant association was found between oral hygiene practice and sex $\mathrm{AOR}=0.636(0.441-0.918, P=0.016)$, indicates females had 
better oral hygiene practice than males ,similar to a study done in Iran [26] in which brushing practice was significant associated with sex $(P=0.006)$, were females had practiced more. Moreover, school type was significantly associated with oral hygiene practice, AOR=0.426, $(0.197-0.92, \mathrm{P}=0.030)$. Bana was more likely to have poor OHP than Arbate Asmara.

\section{CONCLUSION}

This current study revealed that poor level of OHP was prevalent in middle schools of Asmara, Eritrea with discrepancies on how, when and what to use for oral hygiene practice which was alarming for evaluation of the educational program in health institutions, $\mathrm{MOE}, \mathrm{MOH}$ and to incorporate the dental hygiene practices activities by creating linkage and collaboration with health workers and/or institution for efficient and effective health education program to all middle school children periodically. Sex and school type of the middle school children influenced oral hygiene practice in low income countries.

\section{Limitation}

Although this study provides essential information about middle school children in Asmara, Eritrean from the age of 12-15years, it should be interpreted given the limitations presented by a cross-sectional descriptive study and the data for the study was collected from Asmara students enrolled in the middle school, assuming oral hygiene practice would be low in urban than rural . Therefore, this finding might not represent the practice of students out of above study setting or other part of Eritrea, in which their oral hygiene practice might be even better than this study finding.

\section{Implication}

The finding of this study has an implication particularly to Child/Pediatric Nursing \& Dental education, practice, researchers and policymakers of low-income countries. Finally, this study can be used as a baseline to launch further studies regarding children oral hygiene education,

practice and management in the educational sectors and hospitals of Eritrea and other developing countries.

\section{List of abbreviations:}

$\mathrm{OHP}=$ Oral Hygiene Practice, OCMHS= Orotta College of Medicine and Health Sciences, $\mathrm{MOH}=$ Ministry of Health, MOE=Ministry of Education,

SPSS =Statistical Package for Social Sciences, $\mathrm{M} \pm \mathrm{SD}=$ Mean plus/minus Standard Deviation, LMICs= low- and middle-income countries, GNI=gross national income 


\section{Declarations}

\section{Ethics approval and consent to participate}

Ethical clearance was granted from the Ethical Committee of Orotta College of Medicine and Health Sciences and MOH. Permission acquired by directing formal letters and thoroughly explaining the study objectives to school authorities. Confidentiality and anonymity was maintained and written informed consent was obtained from participants' family or guardian that states the data will be used only for statistical purpose. The dignity of participants was maintained and intrusive questions to the participants were avoided.

\section{Consent for publication:}

'Not applicable' for this section

\section{Availability of data and material}

Datasets used to support this study are not publicly available because the ethical approval and consent of participants prohibit sharing of the raw data publicly. However, when deemed necessary, the corresponding author is available to discuss issues regarding data requests.

\section{Competing interests:}

The authors declare that there is no conflict or competing of interest regarding the publication of this paper.

\section{Funding:}

There was no special fund allocated for this study. The Authors conducted the study as part of their employment in the ministry of health and college of health sciences

\section{Authors' contributions:}

"T.O analyzed, interpreted the data and prepared the manuscript regarding the OHP". AH, BM, BT and LH performed the data collection, clearing and entering to SPSS. T.O was a major contributor in writing the manuscript. All authors read and approved the final manuscript."

\section{Acknowledgments:}

We, authors wish to extend our appreciation to the Orotta College of Medicine and Health Sciences, Department of Nursing, Ministry of Health and Ministry of Education. Furthermore, our thanks go Mrs. Paraksamma, who gives us constructive ideas to the research paper development. The authors also wish to thank the school authorities, the children and their parents for their kind participation.

Disclosure: Tecleab Okubai Gebregiorgis is co-first authors. 


\section{References}

1. Bakdash, B., Current patterns of oral hygiene product use and practices. Periodontology 2000, 1995. 8(1): p. 11-14.

2. Petersen, P.E., Global policy for improvement of oral health in the 21 st century-implications to oral health research of World Health Assembly 2007, World Health Organization. Community Dentistry and Oral Epidemiology, 2009. 37(1): p. 1-8.

3. Omale, J.J., Oral Health Knowledge, Attitudes, and Practices Among Secondary School Students in Nigeria. 2014.

4. Grewal, N. and M. Kaur, Status of oral health awareness in Indian children as compared to Western children: A thought provoking situation (a pilot study). Journal of Indian Society of Pedodontics and Preventive Dentistry, 2007. 25(1): p. 15.

5. Mehta, A. and G. Kaur, Oral health-related knowledge, attitude, and practices among 12year-old schoolchildren studying in rural areas of Panchkula, India. Indian Journal of Dental Research, 2012. 23(2): p. 293.

6. Kirk, M.D., et al., World Health Organization estimates of the global and regional disease burden of 22 foodborne bacterial, protozoal, and viral diseases, 2010: a data synthesis. PLoS medicine, 2015. 12(12): p. e1001921.

7. Vishwanathaiah, S., Knowledge, attitudes, and oral health practices of school children in Davangere. International journal of clinical pediatric dentistry, 2016. 9(2): p. 172.

8. Andegiorgish, A.K., et al., Prevalence of dental caries and associated factors among 12 years old students in Eritrea. BMC oral health, 2017. 17(1): p. 1-6.

9. Mudola, J., Factors Influencing Compliance With Oral Hygiene Practices Among Upper Primary School Children In Eldoret Municipality: Uasin Gishu County. 2012.

10. Al-Darwish, M.S., Oral health knowledge, behaviour and practices among school children in Qatar. Dental research journal, 2016. 13(4): p. 342.

11. Organization, W.H., Oral health surveys: basic methods. 2013: World Health Organization.

12. Lateefat, S.a., et al., Knowledge And Practices On Oral Health Among Junior Secondary School Students In Ilorin West Local Government Area Of Nigeria. E-journal of Dentistry, 2012. 2(2).

13. Levin, K.A., Study design III: Cross-sectional studies. Evidence-based dentistry, 2006. 7(1): p. 24-25. 
14. Mlenga, F. and E. Mumghamba, Oral Hygiene Practices, Knowledge, and Self-Reported Dental and Gingival Problems with Rural-Urban Disparities among Primary School children in Lilongwe, Malawi. International Journal of Dentistry, 2021. 2021.

15. Sarwar, A., et al., Oral hygiene practice among the primary school children in selected rural areas of Bangladesh. Journal of Dhaka National Medical College \& Hospital, 2012. 18(1): p. 43-48.

16. Kamran, A., et al., Survey of oral hygiene behaviors, knowledge and attitude among school children: a cross-sectional study from Iran. Int J Health Sci, 2014. 2(2): p. 83-95.

17. McKittrick, T.R. and K.H. Jacobsen, Oral hygiene practices among middle-school students in 44 low-and middle-income countries. International dental journal, 2014. 64(3): p. 164-170.

18. Ndanu, T., et al., Oral Hygiene Practices and Caries Prevalence among 9-15 Years Old Ghanaian School Children. J Nutr Health Sci, 2014. 1(4): p. 406.

19. Priya, M., et al., Oral health attitudes, knowledge and practice among school children in Chennai, India. Journal of education and Ethics in Dentistry, 2013. 3(1): p. 26.

20. Blaggana, A., et al., Oral health knowledge, attitudes and practice behaviour among secondary school children in Chandigarh. Journal of clinical and diagnostic research: JCDR, 2016. 10(10): p. ZC01.

21. Khanal, S. and J. Acharya, Dental caries status and oral health practice among 12-15 year old children in Jorpati, Kathmandu. Nepal Med Coll J, 2014. 16(1): p. 84-87.

22. Burman, J., et al., A study on oral hygiene among students of a secondary school in Harinavi, South 24 Parganas, West Bengal. Medical Journal of Dr. DY Patil Vidyapeeth, 2019. 12(1): p. 34 .

23. Krawczyk, D., et al., Students' knowledge of oral hygiene vs its use in practice. Advances in medical sciences, 2006. 51(1): p. 122-5.

24. Rad, M., A. Shahravan, and A.A. Haghdoost, Oral health knowledge, attitude, and practice in 12-year-old schoolchildren in Iran. Journal of International Society of Preventive \& Community Dentistry, 2015. 5(5): p. 419.

25. Ashok, V.G. and C. Krishnaprasad, A study on oral hygiene among school children in a rural area of Tamilnadu. Int J Contemp Med Res, 2016. 3: p. 2798-9.

26. Ndanu, T., et al., Oral hygiene practices and caries prevalence among 9-15 years old Ghanaian School children. Journal of nutrition and health sciences, 2015. 2(1): p. 1-8.

27. Lian, C.W., et al., Oral health knowledge, attitude and practice among secondary school students in Kuching, Sarawak. Archives of Orofacial Sciences, 2010. 5(1): p. 9-16. 
28. Oyedele, T.A., et al., Social predictors of oral hygiene status in school children from suburban Nigeria. Brazilian oral research, 2019. 33.

29. Elamin, A., M. Garemo, and A. Gardner, Dental caries and their association with socioeconomic characteristics, oral hygiene practices and eating habits among preschool children in Abu Dhabi, United Arab Emirates - the NOPLAS project. BMC oral health, 2018. 18(1): p. 1-9.

30. Halawany, H.S., et al., Effectiveness of oral health education intervention among female primary school children in Riyadh, Saudi Arabia. The Saudi dental journal, 2018. 30(3): p. 190-196.

31. Kuusela, S., E. Honkala, and A. Rimpelä, Toothbrushing frequency between the ages of 12 and 18 years--longitudinal prospective studies of Finnish adolescents. Community dental health, 1996. 13(1): p. 34-39.

32. Kwan, S.Y., et al., Health-promoting schools: an opportunity for oral health promotion. Bulletin of the World Health organization, 2005. 83: p. 677-685.

33. Jones, S., et al., The effective use of fluorides in public health. Bulletin of the World Health Organization, 2005. 83: p. 670-676.

34. Gualie, Y.T. and A.T. Tayachew, Assessment of knowledge, attitude, and practice toward oral hygiene among governmental secondary school students in Debre Tabor Town, Amhara Region, North Central Ethiopia 2018: Institutional-based cross-sectional survey. International Journal of Oral Health Sciences, 2018. 8(2): p. 92.

35. Baral, P., et al., A study on oral hygiene practice among school children of Pokhara Municipality. Journal of GMC-Nepal, 2009. 2(2): p. 37-8.

36. Folayan, M., A. Sowole, and A. Kola-Jebutu, Risk factors for caries in children from southwestern Nigeria. Journal of Clinical Pediatric Dentistry, 2007. 32(2): p. 171-175.

37. Rad, M., A. Shahravan, and A.A. Haghdoost, Oral health knowledge, attitude, and practice in 12-year-old schoolchildren in Iran. Journal of International Society of Preventive \& Community Dentistry, 2015. 5(5): p. 419.

\section{Figure Legends}

Fig.2. Tooth Cleaning Tools Used By the Participants

Fig.1. Frequency of Tooth Cleaning

Fig.3. Prevalence of Tongue Cleaning among Participants 
Fig.4. Frequency of Consumed Sugary Foods and Drinks

Fig.5. Frequency of Dental Visit of the Participants

Fig.6. Reason for Dental Visit among Participants

Fig.7. Status of Oral Hygiene Practice

Table 1: Socio-demographic variables of the children in the study $(\mathrm{N}=572)$

Table 2: Oral Hygiene Practice of Middle School Children

Table 3: Oral Hygiene Practice score difference with Socio-Demographic variables

Table 4: Binary Logistic Regression between Practice and Socio-Demographic Variables 


\section{Figures}

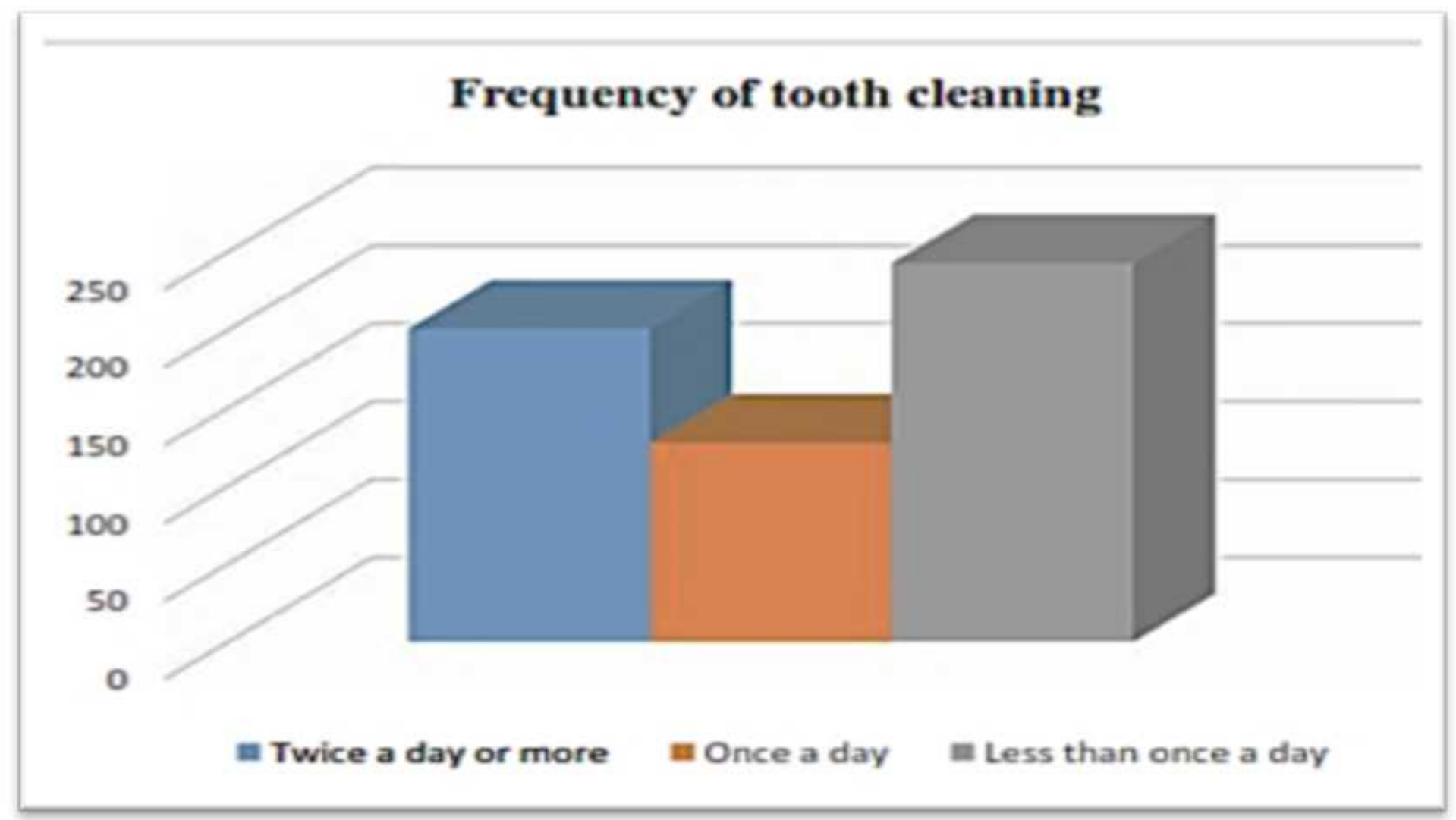

Figure 1

Frequency of Tooth Cleaning

\section{Tooth cleaning tools}

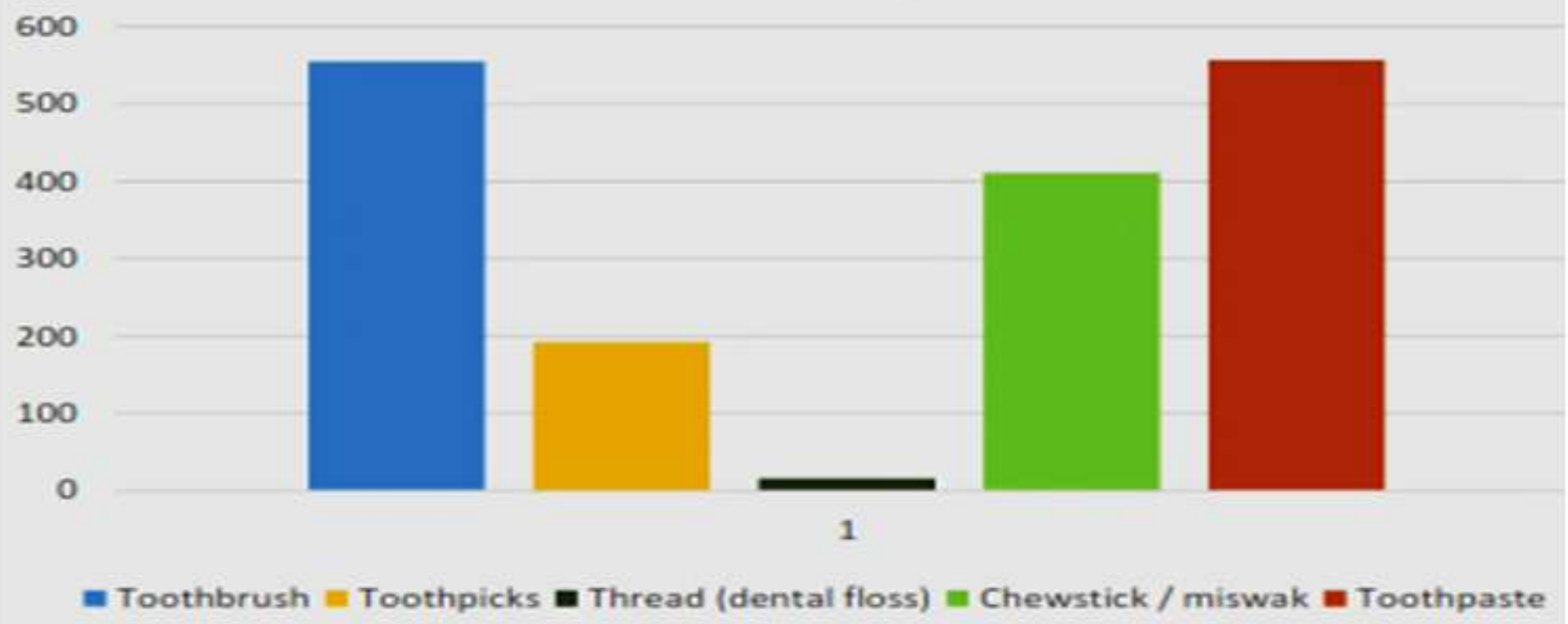


Figure 2

Tooth Cleaning Tools Used By the Participants

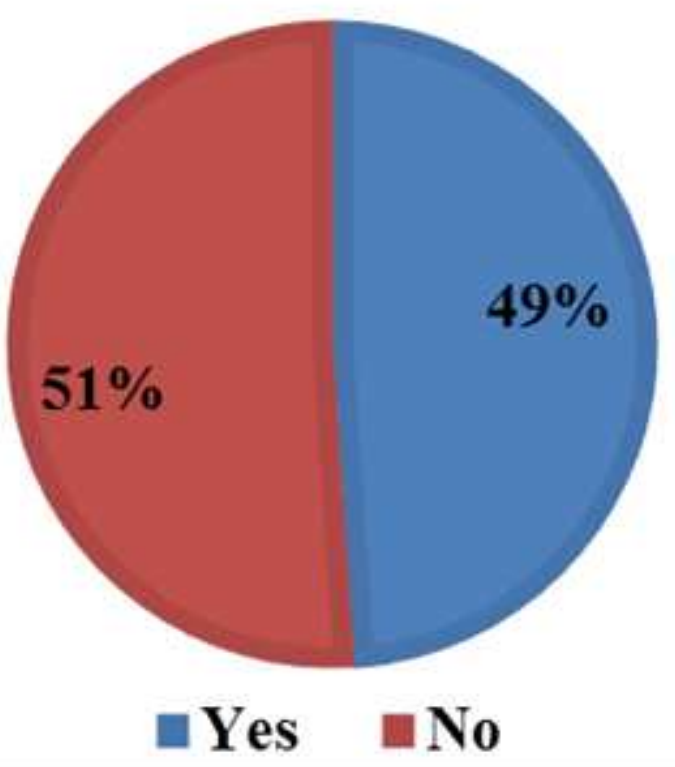

Figure 3

Prevalence of Tongue Cleaning among Participants

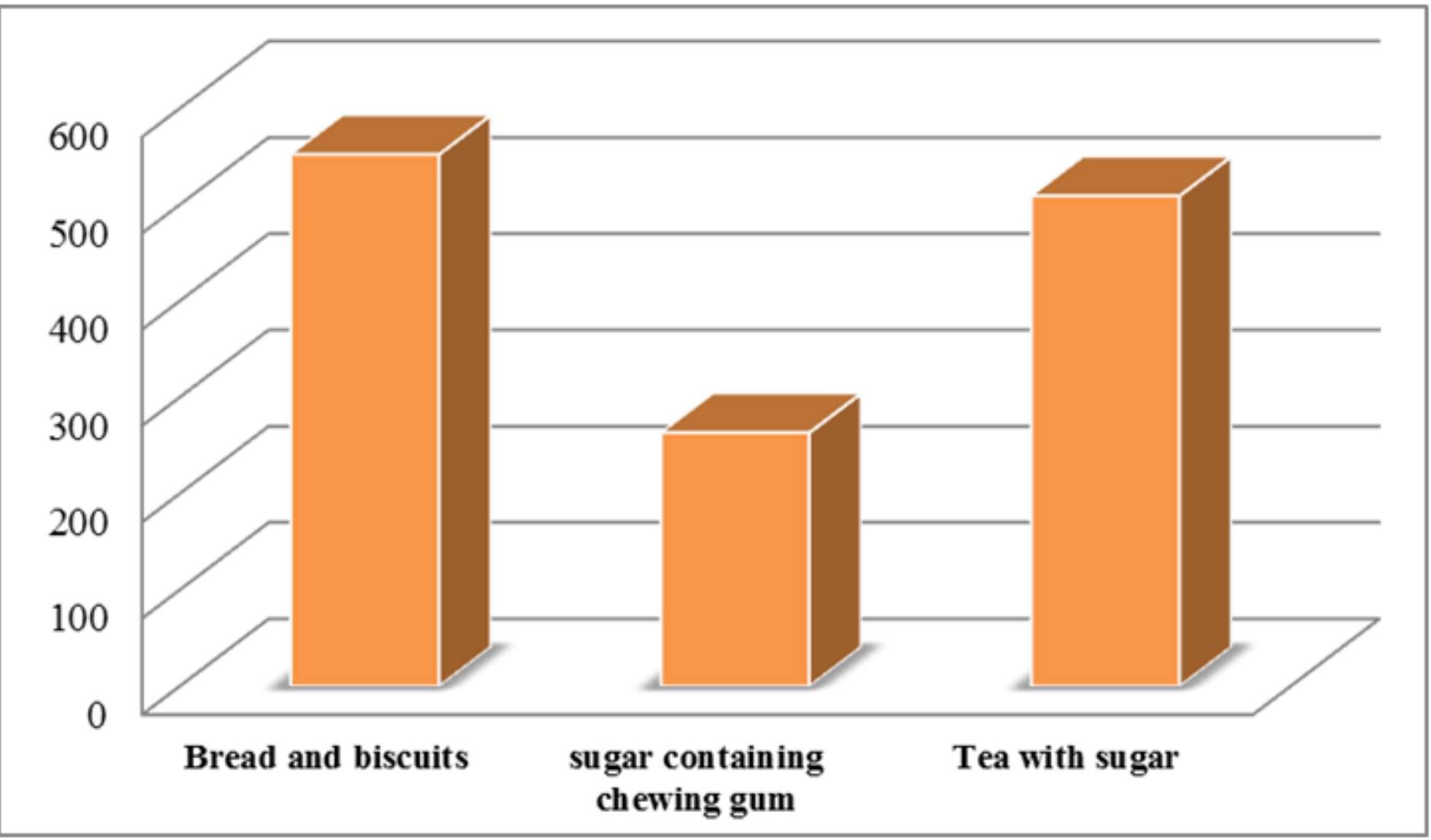


Figure 4

Frequency of Consumed Sugary Foods and Drinks

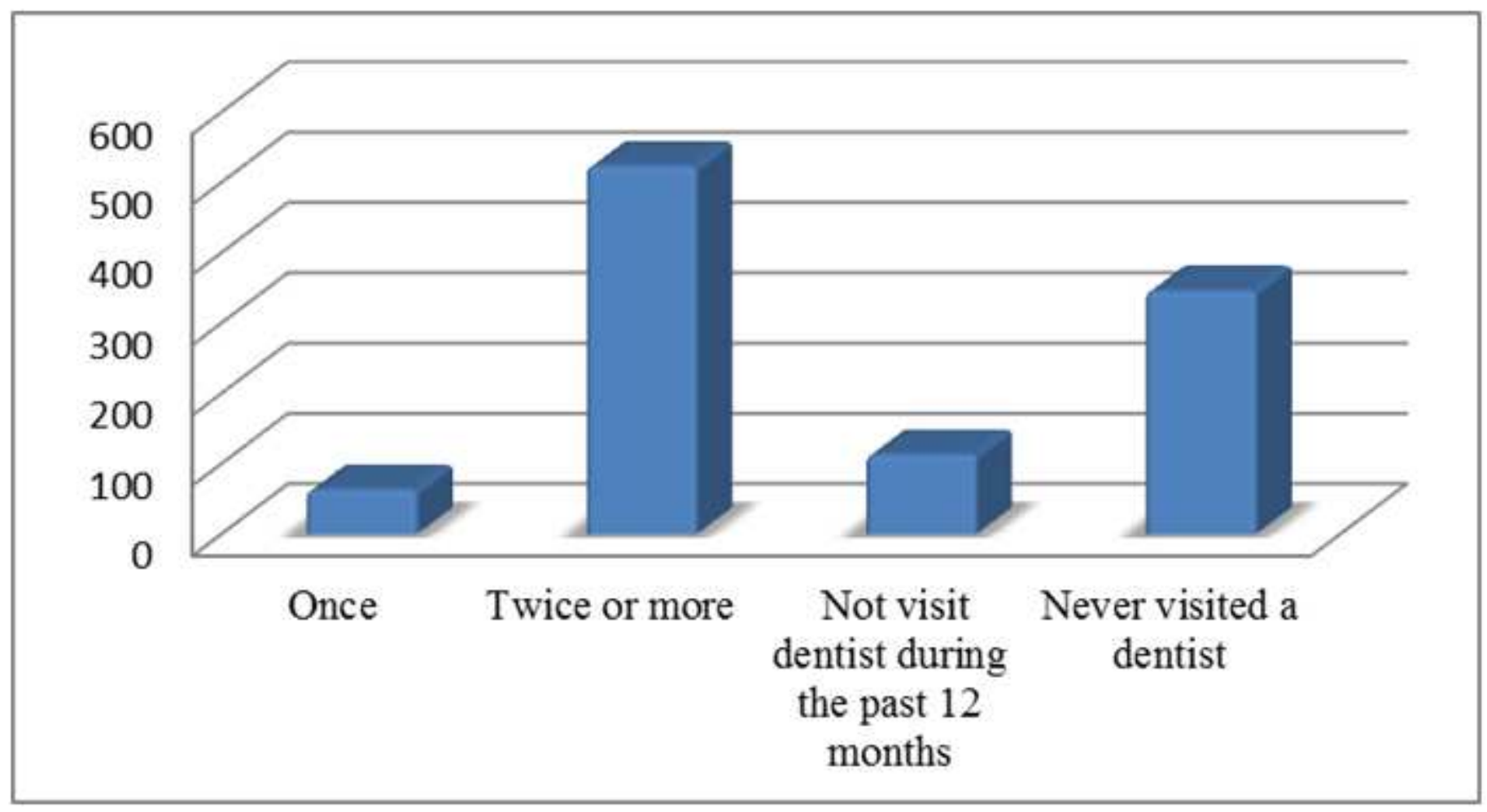

Figure 5

Frequency of Dental Visit of the Participants

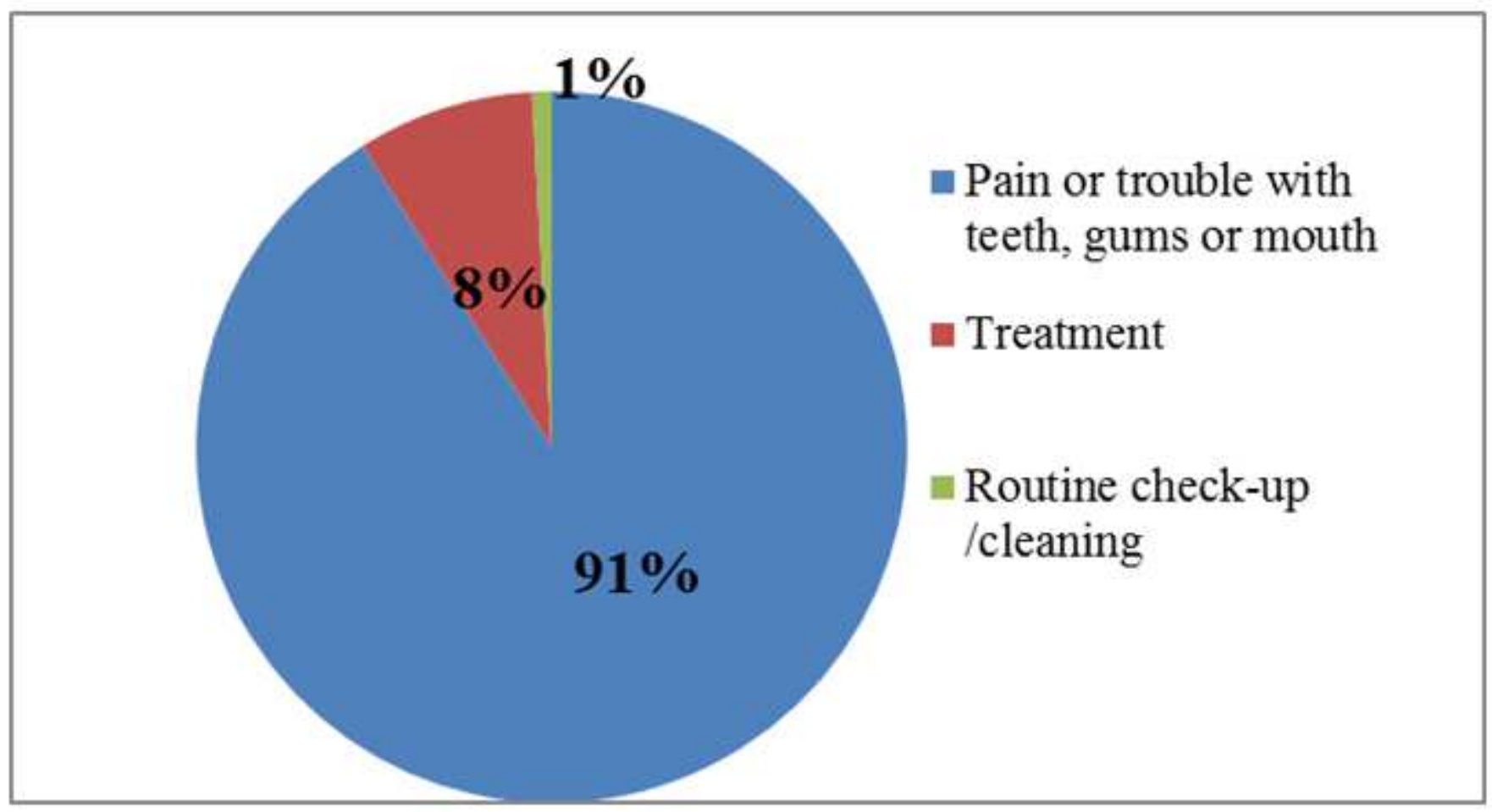

Figure 6 
Reason for Dental Visit among Participants

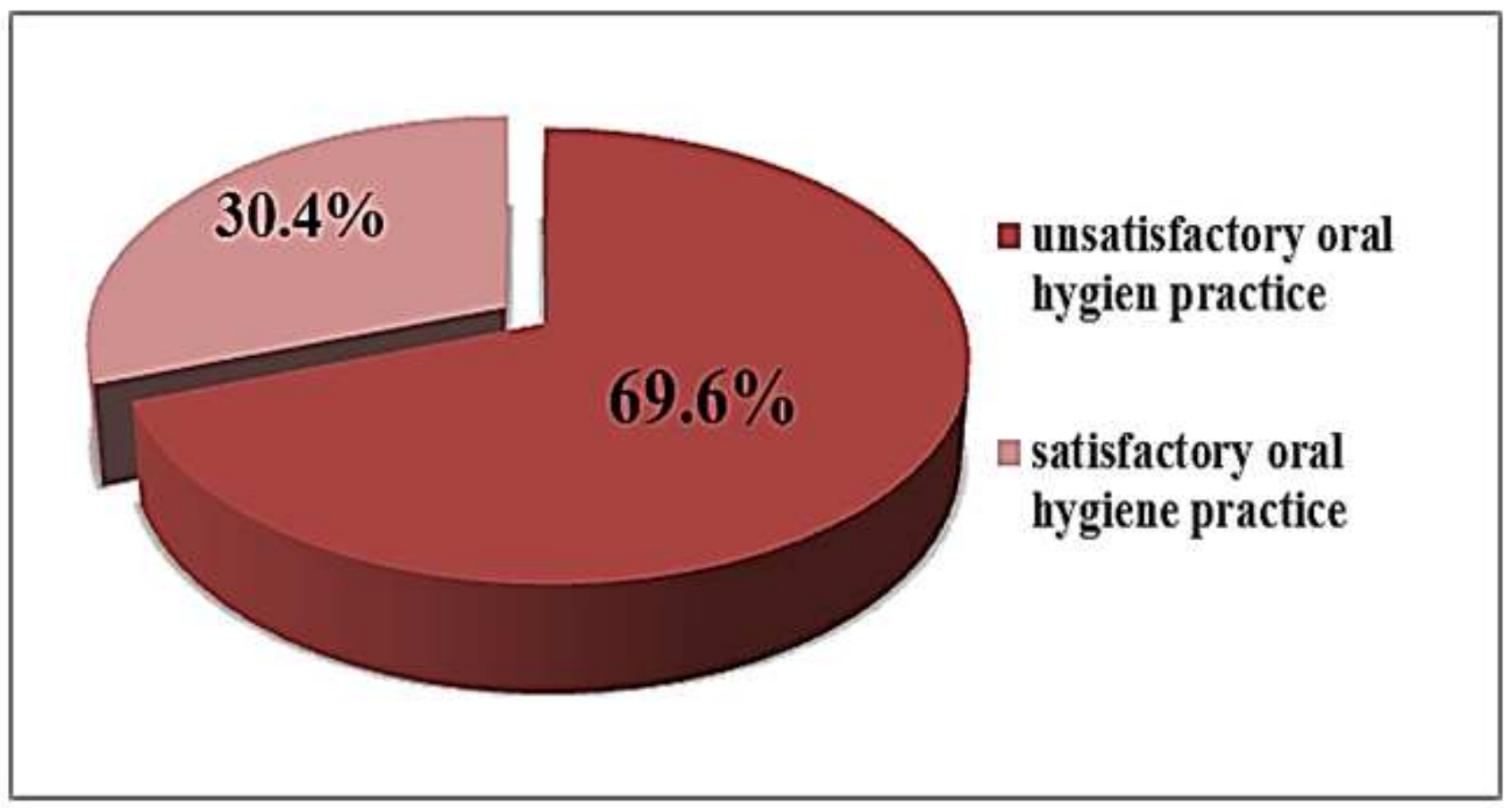

Figure 7

Status of Oral Hygiene Practice 